\title{
LA EDUCACIÓN Y LA ESFERA PÚBLICA: DEL PROYECTO COLECTIVO A LAS INCERTIDUMBRES DEL INDIVIDUALISMO MERCANTILISTA Y DEMOCRÁTICO
}

\author{
José Francisco Durán Vázquez ${ }^{1}$ \\ Universidad de Vigo
}

http://dx.doi.org/10.5209/rev NOMA.2012.v35.n3.42195

\begin{abstract}
Resumen.- El artículo que a continuación se presenta tiene como principal objetivo relatar la crisis de los sistemas educativos actuales en relación con el agotamiento del proyecto civilizatorio moderno amparado e impulsado por el Estado, para el que la educación era uno de sus pilares fundamentales. Para ello se propone en primer lugar un relato diacrónico, en el que se narra la emergencia, el desarrollo y la consolidación de dicho proyecto. Posteriormente, se indican las claves de su agotamiento. A continuación se muestra como los Estados han respondido a esta situación con discursos y con políticas que han erosionado lo esencial de aquél programa civilizatorio, creando un escenario cada vez más individualista y desencantado.
\end{abstract}

Palabras clave.- Estado, educación, civilización, crisis

\section{Education and the public sphere: The collective project to uncertainties of mercantilist individualism and democratic}

\begin{abstract}
The article that later one presents has as principal aim report the crisis of the educational current systems in relation with the exhaustion of the project civilizatorio modern protected and stimulated by the State, for that the education was one of his fundamental pillars. For it one proposes first a statement diachronic, in that is reported the emergency, the development and the consolidation of the above mentioned project. Later, are indicated the keys of his exhaustion. Next it show as the States they have answered to this situation with speeches and with policies that have eroded the essential thing of that one program civilizing, creating a scene increasingly individualistic and disillusioned
\end{abstract}

Keywords.- State, education, civilization, crisis

\footnotetext{
${ }^{1}$ Profesor de Sociología, Universidad de Vigo. Doctor y Licenciado en Sociología por la UNED. Licenciado en Ciencias Políticas y de la Administración, y en Geografía e Historia por la Universidad de Santiago de Compostela. Áreas y temáticas de interés: Sociología del trabajo y del Consumo, Sociología de la Educación, Teoría Sociológica. Especialmente en todo lo referente a la crisis de estos sistemas y a la construcción de nuevas legitimaciones.
} 
Se puede discutir, criticar, rechazar la pedagogía de los colegios del siglo $\mathrm{XIX}$, la de los institutos de 1930 o la de las escuelas primarias de $1880 \ldots \mathrm{El}$ drama es que estos sistemas que caen en la ruina no pueden ser reemplazados por las concepciones demasiado pragmáticas y demasiados funcionales que la sociedad industrial sugiere a la escuela: preparar trabajadores, aprender a aprender, aprender a comunicar, etc. El crecimiento económico y la integración social no pueden disimular por mucho tiempo lo que son: medios a los que la sociedad debe asignar un fin... (Prost, 1967: 494)

\section{INTRODUCCIÓN}

El ámbito educativo ha experimentado importantes transformaciones a lo largo del siglo XX. Durante esta última centuria ha pasado de ser un fenómeno reducido casi exclusivamente a las élites a integrar al común de las gentes. Este proceso conllevó la expansión de los sistemas educativos occidentales, especialmente con posterioridad a la segunda Guerra Mundial, hasta alcanzar unas dimensiones sociales y económicas sin precedentes. Este hecho supuso la realización en buena medida del ideario llustrado, para el que la educación era uno de los principales medios para la realización del espíritu de la época, a saber, una sociedad de individuos autónomos y libres con las mismas oportunidades de desarrollar sus diferentes talentos. Pero este espíritu pronto alcanzó su límite. En efecto, a partir de los años 80 del siglo pasado parecía haber cambiado este escenario. En particular, todos aquellos fines que la modernidad había asignado a la educación eran de cada vez más difícil realización, por lo que fueron criticados desde diferentes frentes. Los sectores progresistas adujeron que los sistemas educativos, lejos de reducir las diferencias sociales, generaban cada vez más desigualdades y eran, además, estructuras autoritarias y represivas. Desde la derecha, se argumentó principalmente que no eran eficientes ni para el mercado laboral ni para la economía.

En este contexto los diferentes Estados iniciaron reformas educativas que han transformado los principios y los fines que habían sostenido el proyecto educativo moderno. Más concretamente, estos principios y estos fines han perdido buena parte de su sentido colectivo para vincularse a los contextos cambiantes de los mercados o a las particularidades de cada individuo. Todo este proceso ha desembocado en una situación en la que la esencia del 
programa civilizatorio moderno, que trataba de integrar la autonomía y la libertad de cada individuo en un universo común de normas y de principios, se ha perdido, y con él buena parte del espíritu que había impulsado la expansión sin precedentes de los sistemas educativos. Se dibuja así un escenario de crisis, por cuanto la mayoría de los actores que integran el ámbito educativo tienen cada vez más dificultades para reconocerse en un determinado proyecto colectivo.

Esta crisis no obedece sólo a la acción de los poderes públicos, en ella están implicadas muchas otras instancias y muchos otros actores. Pero estos poderes con su intervención han contribuido también a producirla. En el artículo que a continuación se presenta se indica el proceso que ha desembocado en la situación actual. Para ello se propone, en una primera parte, un relato diacrónico que parte de la emergencia del pensamiento educativo moderno como proyecto civilizatorio en el marco del Estado Moderno. Continúa comentando la reformulación de este proyecto en el contexto del desarrollo de la sociedad industrial y de la afirmación de los distintos Estados nacionales. Y prosigue narrando su posterior impulso y consolidación, fundamentalmente después de la Segunda Guerra Mundial, por la acción de los Estados democráticos Occidentales. Es la época de la educación de masas. El momento de su plenitud. En la segunda parte se describe el agotamiento de este proyecto a partir de los años ochenta de la pasada centuria. Y como en este contexto los Estados y las principales instituciones políticas internacionales han desarrollado una serie de discursos, que se han traducido en distintas leyes educativas. Leyes y discursos que han contribuido a erosionar buena parte del proyecto educativo moderno, al producir un escenario fuertemente individualizado y desinstitucionalizado, en el que los sistemas educativos carecen de fines claros, la profesión docente se ha deslegitimado, y en el que los alumnos son percibidos como sujetos autónomos, considerados y valorados en los límites de sus particularidades.

\section{1.- ESTADO MODERNO: EDUCACIÓN Y CIVILIDAD}

Parece un contrasentido asociar el Estado Moderno a la educación, pues, como es bien sabido, este Estado jamás intervino directamente en el ámbito educativo, dejando el monopolio de esta materia a las instituciones eclesiásticas (Puelles, 1993). No obstante, si nos referimos a los modelos educativos que surgieron en la Edad Moderna, la influencia del Estado ha sido clave, porque estos modelos, que inspiraron las nuevas formas de educación tanto nobiliares como eclesiásticas, emergieron en el ámbito de una nobleza integrada en las cortes reales o principescas (Elías, 1993a y b; Bowen, 1985, II, pp 307 y ss). Desde esta perspectiva se puede afirmar que, aunque el Estado Moderno permaneció al margen de las instituciones educativas del Antiguo Régimen, creó las circunstancias que originaron unos comportamientos y una sociabilidad que están en la base de un nuevo modo de entender la educación, cuya influencia se dejará sentir a lo largo de la Época Moderna. Este comportamiento y esta sociabilidad respondían a un nuevo concepto de civilidad, definido por las buenas maneras, el refinamiento en el lenguaje, adquirido a través del trato con las gentes de condición noble y la lectura de 
libros clásicos, y un determinado sentido estético. Todos estos aspectos conformarán un nuevo ideal educativo de pretensiones universalistas, que promoverá nuevas formas de socialización y de disciplina orientadas a formar al hombre culto y civilizado.

Con el propósito de inculcar estas actitudes y estos comportamientos, comenzarán a circular en el siglo XVI los libros de urbanidad y de buenas maneras, como el "De civilitate morum puerilium" de Erasmo de Rotterdam (Erasmo, 1985), que tuvo una gran difusión en su época, convirtiéndose en un texto escolar de uso frecuente (Elías, 1993a, pp 99-100; Durkheim, 1992, pp 254; Becci y Julia, 1998, I, pp 292 y ss); o "El libro del cortesano" de Baltasar de Castiglione, más específicamente orientado a mostrar los comportamientos y las actitudes de la nobleza cortesana.

Surgirá así todo un programa educativo de carácter aristocrático-absolutista, inspirado en el mundo clásico greco-latino, con aspiraciones universalistas, que otorgaba una enorme importancia al libro como medio de difusión y de transmisión del saber y de la cultura. Un programa del que surgirán nuevas doctrinas pedagógicas que serán el germen de la moderna pedagogía (Huizinga, 1987: II pp 328; Varela-Álvarez Uría, 1991, pp 56). Esta pedagogía, aunque otorgaba un creciente protagonismo al niño, cuya naturaleza se consideraba que era buena y perfectible, sometía dicha naturaleza a los valores y a las normas de la civilización, fin por antonomasia de toda labor educativa. La educación adquiría así una importancia indiscutible, en cuanto principal instrumento para moldear y perfeccionar el espíritu del niño, liberándolo de su naturaleza más ruda y primitiva, para introducirlo más tarde en las actitudes y los conocimientos del mundo culto y civilizado. Tenía por tanto como uno de sus principales objetivos la intención de socializar, sí, pero también la de construir al sujeto, al sujeto culto y civilizado. Este programa civilizatorio estará vigente hasta el final del antiguo Régimen, cuando la Ilustración lo sustituirá por otro más orientado a saberes más útiles y prácticos, marcando así la transición hacia una nueva sociedad articulada en torno a la industria y el trabajo, y encabezada por un Estado mucho más fuerte y centralizado. No obstante, aunque muchas de las finalidades cambien, permanecerá la intención de integrar, de socializar al individuo, como medio para construir un sujeto. Esta doble condición que tiene lugar en un mismo proceso, el educativo, es la que persistirá más tarde en la llustración, aunque su finalidad ya no sea la misma.

\section{2.- ILUSTRACIÓN Y LIBERALISMO. LA EDUCACIÓN COMO MEDIO DE PROGRESO INDIVIDUAL Y COLECTIVO}

El proyecto civilizatorio cortesano y Humanista fue retomado en las postrimerías del Antiguo Régimen por la Ilustración, pero con otro carácter y con otra finalidad. En efecto, existen importantes diferencias entre el concepto de civilidad, tal como lo habían planteado los Humanistas en el ámbito del Estado Moderno, y el programa educativo que abanderarán los llustrados en el contexto del último Absolutismo. Si bien éstos últimos confían, como antes lo 
hicieran los Humanistas, en la educación como medio de liberación y de transformación de todos los seres humanos, su finalidad es muy otra.

La llustración tiene, en efecto, una intención distinta. No se habla ya de una educación orientada a cultivar los modales y las buenas maneras, la elegancia en el trato o un determinado gusto estético; tampoco se identifica ya la cultura con el aprendizaje de los textos clásicos. En su lugar, surge otra preocupación de carácter más utilitario, más en consonancia con las necesidades del nuevo Estado (Moreno, 1988). Un Estado, que tal como afirmara un famoso Mercantilista a finales del siglo XVIII, "tiene necesidad de hombres- de hombres trabajadores y aplicados-, pero nunca de sabiondos ni de pedantes" (Genovesi, 1785: I, pp 95). Este es el mismo espíritu que anima el código civil Prusiano de 1794, el primer Estado que creó en 1810 un sistema de enseñanza público. En él se establece que "las escuelas y las universidades son instituciones del Estado que tiene por fin la instrucción de la juventud en los conocimientos útiles y científicos" (Luzuriaga, 1994, pp 154). Las materias que deben priorizarse desde este punto de vista son las ciencias y las artes, esto es, los más diversos oficios. Si en este nuevo escenario las letras aún tienen cabida, no será para formar "un espíritu pedantesco, unas ideas abstractas y unas cavilosidades superfluas"; sino para fomentar "un estudio profundo de la naturaleza y unas máximas sólidas dirigidas a la mayor utilidad de los hombres..." (Genovesi, 1785: I, pp 105).

La educación se convertirá por esta vía en una materia de especial interés público, por su contribución a la formación de trabajadores útiles a la república, pero también más capaces e inteligentes. De trabajadores que contribuyan al progreso material de la nación, y también al suyo propio. No obstante, dentro de este marco habrá importantes diferencias entre la instrucción elemental basada en el cálculo y la escritura, dirigida al pueblo, y aquella más refinada, con una mayor presencia de contenidos filosóficos y literarios, reservada a las élites (Varela-Álvarez Uría, 1991, pp 151 y ss).

Esta nueva concepción de la educación alumbrará también una nueva dimensión de la ciudadanía. En efecto, si la educación así entendida contribuía a formar buenos ciudadanos, era porque cada uno de ellos sería capaz de aplicarse con esmero, a partir de las enseñanzas recibidas, en el oficio que hubiese elegido, contribuyendo de este modo a su engrandecimiento y al de la república. Escribe Telleyrand al respecto: "Se debe considerar, en efecto, la sociedad como un vasto taller. No vasta que todos trabajen es necesario que todos estén en su lugar (...) La mayor de todas las economías, ya que es la economía de los hombres, consiste en situarlos en su verdadera posición...es incuestionable que un buen sistema de instrucción es el primero de los medios para lograr esto" (citado en Julia, 1981, p 37). La condición de trabajador y la de ciudadano quedarán así estrechamente vinculadas, y así permanecerán en el futuro (Durán, 2011, pp 245 y ss). No obstante, la ciudadanía no se agotaba en estas enseñanzas utilitarias, imprescindibles para formar parte de la república del trabajo. A ellas habría que añadir también aquellas otras, impulsadas por los emergentes Estados Nacionales, destinadas a crear en el niño la conciencia y el orgullo de su pertenencia a la república. Así se puede leer en un texto del método Peigné destinado a la educación de los niños 
franceses: "cuando seáis hombres ocupareis un lugar en la sociedad. Vuestro primer deber será entonces amar a la patria y obedecer las leyes" (citado en Prost, 1967, p 128). La Geografía y la Historia serán las materias fundamentales para inculcar este sentimiento patriótico en la infancia.

Aunque este espíritu se tiña otras veces de un tono menos patriótico para vincularse con el aprendizaje de las virtudes cívicas de una ciudadanía liberal y democrática. "Cuanto más instruidos sean los hombres- escribe Saint-Étienne durante el proceso revolucionario francés- más conocerán el precio de la libertad y la sabrán conservar; cuanto más luces envuelvan a todos más será mantenida la igualdad entre los hombres (citado en Julia, 1981, p 29).

Durante el siglo XIX y la primera mitad del XX, los profesores se irán empapando de este ideario constitutivo de la nueva "fe laica" republicana (Prost, 1967, p 384). "La escuela- afirma uno de los más destacados representantes del republicanismo francés a finales del siglo XIX- será de ahora en adelante el templo de la fe de los nuevos tiempos. Es misión de los profesores ser los pastores de este templo" (citado en Prost, 1967: 386). La profesión docente adquirirá así el carácter de una especie de sacerdocio que conferirá una elevada autoridad moral a quienes lo desempeñen (Dubet, 2006, pp 40 y ss).

La educación se convertirá de este modo en una materia de vital importancia en la agenda política liberal a lo largo de todo el siglo XIX. "Dejémosla abandonada- dirá uno de sus más destacados representantes- y se verá al instante destruido el nervio más necesario a la conservación y prosperidad del Estado" (citado en Ruíz Berrio, 1970, p 14). Los Estados Nacionales irán construyendo así durante todo este periodo, tanto en Europa como en América, los distintos sistemas educativos, en los que se pretenderá integrar al conjunto de la ciudadanía' (Luzuriaga, 1994: 181, Fernández Enguita, 2001: 45).

\section{3.- LA CONSUMACIÓN DEL PROYECTO REPUBLICANO: LA EXPANSIÓN DE LA EDUCACÓN DE MASAS EN LA SEGUNDA MITAD DEL SIGLO XX}

En las primeras décadas del siglo XX fueron imponiéndose gradualmente las concepciones de la educación más progresistas (Bowen, III 1985, p 550), a través de la difusión del pensamiento de autores como Montessori y Dewey, cuya influencia no sólo fue notable en los gobiernos de sus respectivos países, Italia y EE.UU, sino también en muchos otros de Europa y América, sobre todo en las reformas emprendidas después de la Segunda Guerra Mundial (Manacorda, 1987, p 526). Durante esta época fue aceptándose como un hecho casi indiscutible que la educación era el mejor medio para favorecer el crecimiento económico y el desarrollo social mediante el aprendizaje cultural, cívico y profesional.

Existía también un fuerte consenso acerca de los conocimientos que las instituciones educativas debían proporcionar a los alumnos para atender a todas estas exigencias. Unos se agrupaban en lo que podría denominarse la cultura humanística, de la que formaban parte la geografía y la historia, la 
lengua, la literatura y la filosofía. Otros pertenecían al ámbito de las matemáticas, de las Ciencias Físicas y las Naturales. Y los restantes se integraban en el campo de los saberes éticos y morales. Gozaban de menor consideración, ocupando un lugar secundario en el currículum, los conocimientos de carácter más técnico y profesional.

Existía asimismo consenso entorno al estatuto del alumnado, obligado a asumir una serie de reglas y de normas prescritas como condición necesaria para su aprendizaje.

Había acuerdo también acerca de los conocimientos que debían poseer quienes enseñaban. Los profesores se formaban en las distintas escuelas y facultades, y su titulación, después de haber superado la oposición pertinente, reconocía suficientemente su competencia.

En el ámbito de las realizaciones prácticas, en la primera mitad del siglo XX, los países más industrializados de Europa y de Norteamérica ampliaron sus sistemas educativos hasta abarcar la educación secundaria ${ }^{2}$, aunque con una fuerte dualidad, pues eran minoría los hijos de las clases trabajadores que pasaban por esta etapa educativa (Bowen, III 1985, pp 554 y ss).

En general, se puede decir que a lo largo de este periodo los Estados antes citados destinaron una creciente cantidad de sus presupuestos a la educación, con la intención de instruir a los niños en los conocimientos básicos necesarios para incorporarse al mundo de la industria.

No obstante, la gran expansión educativa no se produjo en los países occidentales hasta después de la Segunda Guerra Mundial (Prats-Reventós, 2005, p 230). Pasada la primera década después de la Gran Guerra, en la que los países europeos fueron recuperándose poco a poco de la ruinosa situación en la que habían quedado sumidos, se inició una etapa histórica en la que se dedicaron enormes esfuerzos al campo educativo hasta niveles presupuestarios nunca antes vistos. Los motivos que estaban detrás de esta orientación eran sociales, políticos y económicos. Desde el punto de vista social y político, la educación fue considerada como el principal mecanismo de integración y de progreso social, el mejor modo de hacer realidad el principio meritocrático en una sociedad democrática e igualitaria. Cómo afirmaba el sociólogo T.H Marshall a comienzos de los años cincuenta, haciéndose eco del sentir de la época, se trataba de "permitir que el niño pobre demuestre ser tan capaz como el rico". "La consecuencia final-concluía- es una estructura de estatus desigual ajustada a las capacidades desiguales" (Marshall, 1998, p 67). Desde la perspectiva económica, la educación fue percibida como uno de los motores principales del crecimiento económico, cobrando fuerza, a partir de los años sesenta especialmente, la noción de la educación para el desarrollo (Ortega, 1993, pp 89 y ss; Rodríguez, 1988; Bowen, 1985 III, p 655). Se entendía, desde esta óptica, que la educación era un factor clave de la economía. En este ambiente emergió dentro de la ciencia económica la conocida como Teoría del Capital Humano. Con arreglo a esta teoría, se consideró que existía una relación positiva entre el número de personas cualificadas y el progreso económico nacional, y que se precisarían cada vez 
más personas con estudios para atender a la demanda de unas economías en constante crecimiento. A este fin los principales organismos internacionales, tales como el Banco Mundial o la OCDE, hicieron recomendaciones a los Estados para que incrementasen sus presupuestos educativos (Rodríguez, 1988). Para dar respuestas a estas inquietudes los gobiernos iniciaron ambiciosos programas de planificación educativa a los que destinaron ingentes recursos ${ }^{3}$ (OCDE, 1991, p 13). Dichos programas conllevaron las reformas de los sistemas educativos de la mayoría de los países europeos occidentales, que incluían la universalización efectiva y la prolongación de la enseñanza hasta abarcar las etapas iniciales de la educación secundaria ${ }^{4}$ (Taberner, 1999).

El consenso de postguerra acerca de las potencialidades políticas, sociales y económicas de la educación, no sólo permitió, como se ha visto, un crecimiento incomparable de los sistemas educativos, posibilitó asimismo que los valores que estaban detrás de este programa institucional (Dubet, 2006), impulsado y sostenido por los distintos Estados, fuese ampliamente incorporado por la mayoría de la población como el medio principal para el desarrollo y la liberación de los sujetos. Se puede decir, por tanto, que la implementación de dicho programa contribuyó en buena medida a la legitimación de los Estados que lo pusieron en práctica.

\section{4.- LA CRISIS DEL PROGRAMA REPUBLICANO}

En los años 70 las causas que habían favorecido el desarrollo de la educación de masas después de la Segunda Guerra Mundial, se habían modificado. El crecimiento económico continuado que había caracterizado las casi tres décadas posteriores a la gran Guerra, y que inauguró lo que se conoce como sociedad de consumo fordista ${ }^{5}$, parecía agotado, y con el también el optimismo que lo había acompañado (Piore y Sabel, 1990; Alonso, 2005). Por lo que a la educación se refiere, la asociación tan estrecha que se había establecido entre cualificación y empleo en la época dorada de la expansión económica, y que justificó el desarrollo de la educación de masas, ya no gozaba del mismo predicamento. La obtención de un diploma ya no facilitaba como antes el acceso al mundo laboral, y si lo hacía era en unas condiciones mucho más desfavorables. Aunque las cualificaciones eran cada vez más demandadas, su posesión ya no garantizaba la consecución de un empleo (Serrano Pascual, 2000). De forma paradójica, los títulos académicos se devaluaban justo en el momento en que eran también más solicitados (Beck, 2006, pp 241 y ss). Todo esto significaba que las instituciones educativas estaban perdiendo su capacidad anterior para asignar legítima y eficientemente posiciones sociales de acuerdo con los principios de mérito y capacidad, principios que estaban en la base de todo el ideario educativo posterior a la llustración (Habermas, 1999, pp 141-42).

Desde este punto de vista, la crisis educativa a la que acabamos de referimos no consistía solamente en un problema de adecuación entre las instituciones educativas y los mercados laborales. Implicaba también el cuestionamiento de la capacidad que tenían aquellas instituciones para cumplir eficientemente con 
el programa llustrado en todas sus vertientes. Y este cuestionamiento procedía de todos los sectores ideológicos. En el ámbito de la izquierda aparecieron en los años setenta toda una serie de estudios que destacaban el carácter reproductivo y represivo de los sistemas educativos. Todos ellos alcanzaron una gran difusión, y se convirtieron rápidamente en obras de gran referencia. Merecen ser destacados desde este punto de vista los trabajos de Althusser (Althusser, 1974, pp 97 y ss); Bourdieu (Bourdieu-Passeron, 2001); Baudelot y Establet (Baudelot-Establet, 1975); Bernstein (Bernstein, 1989), Alexander (Alexander, 1970); Paul Willis (Willis, 1988) y Foucault (Foucault, 1999). Todos coincidían en criticar las estructuras jerárquicas y autoritarias que articulaban el mundo educativo, y también el proceso de transmisión del conocimiento, así como el conjunto de saberes que a él iban asociados (Le Goff, 2002, p 98).

Otros escritos pusieron en tela de juicio la igualdad de oportunidades educativas. En EE.UU alcanzó gran difusión el Informe Coleman y las investigaciones de Christopher Jencks, que cuestionaban abiertamente que la educación pudiese contribuir realmente, tal como se creía, a la igualdad y a la democracia (Bowen, 1985 III, p 676).

Fue así extendiéndose la idea, sobre todo entre los intelectuales de izquierdas y entre la población instruida vinculada a dichas corrientes, de que los sistemas educativos no sólo no lograban el objetivo proclamado de democratización e igualación entre las clases, sino que eran, además, instituciones eminentemente alienantes y represivas.

El escepticismo educativo fue también en aumento en el ámbito del liberalismo, sobre todo a raíz de la crisis de mediados de los años setenta. Esta crisis puso de manifiesto que la creciente oferta educativa que se había producido en los países occidentales en el periodo anterior, no podía ser absorbida por unas economías cada vez más desaceleradas (Coombs, 1985, pp 29 y ss). Se imponía, por tanto, la reforma de estas instituciones para adecuarlas eficientemente al mercado laboral.

En suma, para los sectores progresistas la educación era un sistema netamente desigualitario y represivo, y para los liberales una actividad que no generaba la rentabilidad económica esperada, y que producía, además, cada vez más paro. Las razones políticas, económicas y sociales que habían impulsado el enorme crecimiento de los sistemas educativos después de la segunda Guerra mundial parecían ahora desacreditadas.

En este nuevo contexto se propusieron distintas soluciones. Los Liberales, optaron por recetas de eficiencia económica, adecuando la enseñanza al sistema productivo. Se plantearon medidas como reformar el currículum educativo, capacitar a los profesores o mejorar la gestión educativa (Rodríguez, 1988, Terren, 1999, pp 215-16; Prats-Reventós, 2005, p 17) ${ }^{6}$. La palabra más utilizada en este contexto fue la de calidad de la enseñanza (OCDE, 1991, pp 27 y ss). Todas estas medidas estuvieron en boga durante los años ochenta como parte del programa de los gobiernos neoliberales, y continúan estándolo hoy en día. Desde el lado socialdemócrata, por el contrario, se hacía más hincapié en las políticas democratizadoras, sin que ello signifique que se obviasen los principios meritocráticos y economicistas, y también la formación 
del profesorado. Desde este ámbito se abogaba por diversificar el proceso educativo, para tener así en cuenta la realidad de cada alumno ${ }^{7}$ (Prost, 1967, p 492; Enkvist, 2000, pp 232 y ss).

Los alumnos, en efecto, pasaron a tener un mayor protagonismo en la esfera educativa. A este hecho colaboraron factores tales como el ambiente intelectual creado por aquellas obras aparecidas en los años 70, para las que los sistemas de enseñanza eran auténticos escenarios alienantes y reproductivos (Torrés Santomé, 2008, pp 144 y ss), un proceso progresivo de ampliación de los derechos del niño y de su inclusión en la ciudadanía (Renaut, 2002; GaitánLiebel, 2011), y también una concepción de la enseñanza que hacía más hincapié en el método que en el contenido, y que en consecuencia estaba más preocupada por el aprendizaje que por la transmisión del conocimiento.

En suma, en las últimas décadas del siglo pasado se fue dibujando un escenario que iba a producir cambios sustanciales en muchos de los sistemas educativos de los países occidentales. Por una parte, fueron decreciendo las esperanzas acerca de la capacidad de estos sistemas para producir mayor igualdad y democracia, al tiempo que eran percibidos como más opresivos y autoritarios. Por la otra, el aumento de los niveles de desempleo entre la población cualificada generó una serie de respuestas, consistentes en mejorar las formas de gestión, reformar el curriculum y proponer más formación para los profesores.

En este contexto se fueron erosionando muchos de los principios que hasta ese momento habían legitimado y sostenido la acción educativa. En particular, resultaron afectados los dos polos de dicha relación. Desde el lado de la enseñanza, todo lo relacionado con la concepción de los saberes y el proceso de transmisión de los mismos. En efecto, ¿qué contenidos serían los más adecuados para unos sistemas educativos que se alejaban progresivamente del mundo del trabajo, y que eran percibidos además como crecientemente opresivos, desigualitarios e injustos? En relación con el aprendizaje, se hizo cada vez más hincapié en el alumno y sus capacidades en unos contextos cada vez más abiertos, participativos y democráticos. Se produjo así una reformulación de los procesos de enseñanza-aprendizaje en el marco de los discursos elaborados por las instituciones políticas y por los saberes pedagógicos y psicológicos (Raynaud, 1983, p 118).

Todo este proceso desembocará, como luego veremos, en una creciente desinstitucionalización e individualización de los procesos de enseñanzaaprendizaje, que acentuó más todavía la crisis de los sistemas educativos. Todas estas cuestiones serán abordadas en el próximo epígrafe.

\section{5.- LA REFORMULACIÓN DEL PROYECTO REPUBLICANO}

\section{1.- Una nueva concepción de los saberes}

Como se ha señalado anteriormente, una de las manifestaciones de la crisis educativa actual es la incapacidad que tienen los sistemas de enseñanza para 
generar empleo. Esta situación, que viene ya produciéndose desde los años 80 de la pasada centuria, es una cuestión de enorme importancia porque afecta a uno de los aspectos esenciales del ideario educativo moderno. En efecto, la educación emergió a lo largo de la modernidad vinculada al mundo del trabajo como instrumento principal para la realización del espíritu de la época, a saber, una sociedad de individuos libres con las mismas oportunidades de promoción y de ascenso social. Quizás fue el sociólogo T.H Marshall, a quien hemos citado anteriormente, quien mejor resumió este espíritu. "Por mi parte- escribió a comienzos de la década de los 50- no encuentro rastros de relajación de los vínculos que unen la educación a la ocupación. Por el contrario, parece que se estrechan cada vez más, porque aumenta el respecto por los certificados, los diplomas y las licenciaturas en la medida en que sirven como cualificaciones para un puesto de trabajo, y no parece que la tendencia disminuya con el paso del tiempo" (Marshall, 1998, p 66)

Durante las tres décadas posteriores a la segunda Guerra Mundial este optimismo no careció de sentido, ya que los diplomas académicos que otorgaban las instituciones educativas gozaban de amplia aceptación y reconocimiento en el mundo profesional, confiriendo posiciones y prestigio (Laval, 2004, p 51).

Esta situación es la que más ha cambiado tras las tres décadas de expansión económica y educativa sin precedentes posteriores a la gran Guerra. A partir de ese momento, las cifras de desempleo comenzaron a incrementarse entre la población cualificada (Serrano, 2000: 137; OCDE, 1992, p 11; Alaluf, Stroobants, 1994, p 48). Si bien las credenciales educativas continúan siendo un requisito necesario para acceder al mercado de trabajo, este requisito ya no es suficiente (Miguelez y Prieto, 1999, p 188; Beck, 2006, pp 241 y ss). "Para el relanzamiento del crecimiento, la restauración de la competitividad y el establecimiento de un nivel de empleo socialmente aceptable en la Comunidadse comentaba en el Libro Blanco de la Comisión para las Comunidades Europeas-, la educación y la formación, al tiempo que mantienen su misión fundamental de promover el desarrollo personal y los valores de la sociedad, tendrán, sin duda, que desempeñar un papel determinante. No obstante- se aclara- conviene comprender su carácter, alcance y límites" (Comisión de las Comunidades Europeas, 1993, p 126).

El hecho de que la educación y la formación se planteen como algo necesario pero ya no suficiente, está directamente relacionado con las transformaciones que experimentó el modelo productivo fordista a partir de los años 70 del siglo pasado, como consecuencia del agotamiento de los mercados de masas. Para reaccionar a esta situación, se diseñaron nuevas estrategias empresariales consistentes en flexibilizar la producción adaptándola a los cambios en la demanda. Para ello se hicieron importantes inversiones en tecnología para abaratar costes, y diversificar y mejorar la calidad de los productos (Piore y Sabel, 1990, pp 263 y ss). Como consecuencia de todas estas transformaciones, el nuevo sistema productivo demandó otro tipo de formación en el que las cualificaciones educativas eran necesarias pero ya no suficientes. De este hecho eran conscientes no sólo las organizaciones empresariales, sino también las instituciones políticas internacionales más importantes. Así se 
podía leer a comienzos de los años 90 en un Informe de uno de estos organismos: "el reproche esencial que se hace a la enseñanza- decía- es que las instituciones académicas dispensan cada vez menos conocimientos y cualificaciones necesarias para cuando los alumnos comiencen a trabajar" (OCDE, 1992, pp 9-10). Se precisaban otro tipo de cualidades más adaptadas a cada estructura productiva, de carácter menos formal y más subjetivo. "Los jóvenes que abandonan la escuela- afirmaba otro de estos informes- tienen necesidad de una gama de cualificaciones y de competencias que sólo en parte se adquieren en un programa tradicional de estudios" (OCDE, 1998, pp 25-39). Estas cualidades, agrupadas en el concepto de competencias, "exigidas en el mundo del trabajo, que las empresas quieren fomentar cada vez más que se enseñen en las escuelas, son de orden más general. La adaptabilidad, la facultad de comunicar, de trabajar en equipo, de tener espíritu de iniciativa, estas cualidades y otras competencias "genéricas"- señalan estas instituciones- son ahora esenciales para asegurar la competitividad de las empresas". Habrá, por tanto, que "desarrollar- aconsejan los miembros de estos organismos- una pedagogía nueva en la enseñanza general para formar trabajadores más adaptables y más reflexivos de los que los empleadores tienen hoy necesidad" (OCDE, 1992, pp 7-11, comillas del original)

No obstante, el nuevo escenario formativo y educativo que surgió a partir de finales de los años 70 no respondía únicamente a una mera adaptación de los sistemas educativos a las nuevas circunstancias productivas. En efecto, no sólo se trataba de atender a los nuevos requerimientos del sistema económico, había que prestar también atención a los imperativos del sistema político, fortaleciendo la legitimidad del Estado, y del sistema cultural, manteniendo una actitud favorable hacia el empleo de quienes se forman, para que así percibiesen su proceso de formación como una etapa previa a su incorporación al mundo del empleo. Para ello había que crear una nueva simbología que vinculase la educación con el empleo de otro modo, porque ambos fenómenos son parte esencial de la estructura de legitimaciones de las sociedades modernas. En palabras de Bourdieu, había que dar a los nuevos discursos la forma reconocida como conveniente, pues presentada de otro modo sería considerada inaceptable (citado en Ropé-Tanguy, 1994, p 239). En un contexto como el postfordista de pérdida del valor de las cualificaciones, la relación entre educación y empleo tenía, por ello, que formularse en un lenguaje que fuese más atractivo para los mercados de trabajo, más legitimador para los Estados, pero también más motivador y más movilizador para los sujetos (Boltanski y Chiapello, 2002, p 59).

Los informes y las reformas que las instituciones políticas nacionales e internacionales vienen promoviendo, fundamentalmente a partir de la década de los años 80 del siglo pasado, tienen precisamente estos objetivos. En dichos textos el concepto de cualificación pierde peso en favor de la noción de competencia, más próxima a lo que demandan los mercados laborales, más pertinente para la motivación de los sujetos, y más necesaria también para la legitimación de los Estados.

En los discursos que estructuran dichos textos sigue reforzándose por ello, quizás más que nunca debido a su debilidad, la relación entre formación y 
empleo, pero apelando a un nuevo concepto de formación que va mucho más allá de los títulos académicos propiamente dichos. "Sería deseable", se afirma en uno de estos documentos políticos, "que los alumnos aprendan a aprender en lugar de aprender para tener un diploma" (OCDE, 2000, p 34). El contenido de este nuevo aprendizaje incluiría, por tanto, una serie de habilidades, tales como "ciertas disposiciones, aptitudes y competencias del individuo: creatividad, iniciativa, aptitud para la resolución de problemas, flexibilidad, capacidad de adaptación, responsabilidad, aptitudes de aprendizaje y reciclaje" (OCDE, 1992, p 9). Y, por encima de todo, "la capacidad fundamental de adquirir nuevos conocimientos y nuevas competencias, "de aprender a aprender" a lo largo de toda la vida" (Comisión de las Comunidades Europeas, 1993: 130, comillas del original). De acuerdo con todas estas concepciones, la OCDE ha elaborado en el año 2000 un documento en el que se definen y seleccionan las competencias clave que han de ser enseñadas y aprendidas, el denominado proyecto DeSeCo (Definición y Selección de Competencias Clave) (Rychen y Salganik, 2004). Estas competencias tratan de superar un enfoque excesivamente academicista de la educación, para privilegiar otro más práctico y funcional, orientado a "hacer frente a situaciones de la vida", esto es, a "la adquisición de capacidades para enfrentar al mundo económico o profesional, resolver problemas sociales, etc..." (Rychen y Salganik, 2004, p 21; Bolivar, 2008, p 12). Todo ello desde una perspectiva más focalizada en el aprendizaje que en la enseñanza. Pues "la mayoría de las competencias- tal como dice el proyecto DeSeCo- se relacionan con el aprendizaje". Un aprendizaje encaminado, en un mundo cambiante e incierto como el actual, no tanto a adquirir unos determinados contenidos como a desarrollar capacidades para que los jóvenes sepan "manejar situaciones imprevistas" (Rychen y Salganik, 2004, p 22; Bolivar, 2008, p 4). Las competencias se centran por tanto más en el hacer que en el conocer. Se relacionan más con la intervención práctica que con la asimilación de contenidos. "La cultura- afirman los defensores de este discurso- tiene que percibirse como útil, operativa por parte de los estudiantes. El conocimiento como valor de uso" (Pérez Gómez, 2008, p 88). Por último, las competencias están más orientadas a incentivar y a estimular el aprendizaje del alumno que a la transmisión de conocimientos por parte del profesor.

La nueva cultura de las competencias se hermana por ello perfectamente con las nuevas tecnologías. En efecto, una concepción del saber más preocupada por inculcar habilidades y capacidades que por transmitir conocimientos, más aferrada al método que al contenido, y más atenta a los contextos cambiantes de los nuevos escenarios productivos, confía plenamente en las posibilidades ofrecidas por las nuevas tecnologías. Esta obsesión por las tecnologías es evidente desde hace dos décadas en los discursos de los organismos Internacionales, tales como la OCDE o la UE. Desde esta última institución se vine estimulando insistentemente la investigación en "programas informáticos educativos multimedia" (Laval, 2004, p 178). La tecnología ha pasado a ocupar también un lugar destacado en los nuevos programas escolares. En 1997 Tony Blair lanzaba un ambicioso plan informático para las escuelas británicas. En ese mismo año el gobierno de Lionel Jospin proponía medidas similares para la educación francesa (Laval, 2004, p 179). En España se han puesto en marcha un plan ambicioso para dotar a los centros educativos de material informático a disposición de los alumnos. 
Para poner en práctica este nuevo programa educativo, afirman los representantes de estas instituciones y organismos internacionales, "se hace indispensable una renovación de la organización de la escuela y de los contenidos y métodos pedagógicos" (OCDE, 2000, p 34). Y esto es precisamente lo que viene ocurriendo desde la década de los 80 cuando muchos de los conceptos que incluye el nuevo programa educativo han impregnado las reformas y los reglamentos de muchos Estados (Bolivar, 2008, pp 2-3). Estos documentos vienen refiriéndose cada vez con más fuerza a la noción de competencias como un concepto subjetivo centrado en una serie de habilidades y capacidades que el alumno debe poner en práctica en situaciones concretas de aprendizaje. En Francia este escenario viene dibujándose desde la Reforma de 1989 (Le Goff, 1999: 37 y ss), en España desde que lo incorporó la LOE en 2005.

En este contexto ha emergido con gran fuerza el constructivismo pedagógico que otorga un gran protagonismo en los procesos de educativos al método y al aprendizaje experimental antes que a los contenidos propiamente dichos (Laval, 2004, p 182; Torres Santomé, 2008, p 165).

En suma, el discurso de las competencias con una concepción de la enseñanza eminentemente utilitarista y práctica, focalizado más en el aprendizaje que en la enseñanza y orientado hacia el presente, contribuye sin duda a socavar las nociones anteriores del saber y de la cultura, basadas en una herencia común que las instituciones educativas transmitían por medio de sus representantes, los profesores (Le Goff, 1999, p 44). Esta herencia común, con todo el conjunto de saberes que la integraban, está siendo sustituida por una serie de procedimientos y de métodos adaptados a la particularidad de cada contexto. Todo ello está contribuyendo a la desinstitucionalización y a la individualización de los procesos de enseñanza.

\section{2.- El protagonismo del alumno y la cultura del aprendizaje}

A esta desinstitucionalización ha contribuido también la tendencia de los actuales discursos educativos a centrarse en el alumno. Esta situación no ha emergido espontáneamente, tiene raíces profundas. En efecto, el discurso pedagógico y psicológico, tal como se ha configurado a lo largo de la época moderna, esta articulado alrededor del niño (Raynaud, 1983, p 119; Renaut, 2002; Gauchet, 2002, pp 123-24), por lo que no es extraño que tanto los psicólogos como los pedagogos que amparan muchas de las actuales reformas educativas defiendan esta perspectiva. Pero su vigencia actual no se debe sólo a este hecho. El protagonismo que ha adquirido el alumno en los sistemas educativos viene acentuándose especialmente desde los años 70 de la pasada centuria. Confluyen en ello varias circunstancias. Por un lado, las críticas vertidas hacía unos sistemas de enseñanza considerados opresivos e injustos, puso en cuestión los contenidos del curriculum tradicional en favor de los intereses y las particularidades del niño (Dubet, 2006, p 89). La sociología, la psicología y la pedagogía fueron las disciplinas que más legitimaron este discurso (Dubet, 2006, p 108; Torrés Santomé, 2008, pp 145-146). 
Por el otro, el desarrollo de un movimiento, enraizado en la cultura moderna, que aboga por la progresiva emancipación y la autonomía del niño (Arendt 2003, p 289; Therborn, 1993, p 115), y que ha desembocado finalmente en su inclusión en la esfera pública como un verdadero ciudadano. Las consecuencias de este proceso fueron especialmente perceptibles con posterioridad a la Segunda Guerra Mundial, aunque en los países nórdicos, particularmente en Noruega y en suecia, comienzan ya a ser visibles en los años 10 y 20 del pasado siglo. En el otro extremo se encuentran los países del sur de Europa, tales como España, Portugal o Grecia, que no se incorporaron a esta tendencia hasta la década de los 80 del siglo XX (Therborn, 1993, pp 99 y ss). Este proceso se formalizó por primera vez internacionalmente en la Convención de los derechos del niño, aprobada por la Asamblea de Naciones Unidas el 22 de noviembre de 1989, en la que se reconocían al niño una serie de derechos, antes sólo privativos de los adultos. Entre estos derechos estaban los de libertad de opinión, de expresión, de pensamiento, de conciencia, de religión, de asociación, de reunión pacífica, e incluso el derecho a que se respetase su vida privada (Rénaut, 2002, pp 375 y ss; Gaitán, 1999, pp 71 y ss; Gaitán y Manfred, 2011, p 29). En esta misma dirección, el Consejo de Europa en su asamblea de 1996 propuso a todos los Estados miembros la suscripción de un acuerdo para reconocer los derechos de la infancia, en cuyo punto 5 se afirmaba que "el respeto por los derechos de la infancia y una mayor igualdad entre niños y adultos ayudaría a preservar el pacto entre generaciones y contribuiría a la democracia" (Citado en Rodríguez Pascual, 2007, p 125).

Todos estos acuerdos, cuya ratificación tenía carácter vinculante para los Estados firmantes, supusieron un avance significativo en los derechos de los niños. En efecto, a partir de ese momento la sociedad y los poderes públicos no sólo reconocían la obligación de protegerlos en virtud de su inmadurez y debilidad, protección necesaria por otra parte para que lleguen a ser algún día adultos libres y responsables, sino que, además, les otorgaban, en tanto que otros sujetos con derechos de ciudadanía, un estatus semejante al de los adultos (Rénaut, 2002, pp 393 y ss). La infancia entraba así por la puerta grande en el espacio público democrático, culminando un proceso que primero había emancipado a los trabajadores, después a las mujeres y por último a los niños (Therborn, 1993, p 96).

A este nuevo estatuto de la infancia no serán ajenas tampoco las leyes educativas, que concederán al alumno un papel mucho más activo y participativo, por ser también ellos sujetos de derechos (Therborn, 1993, pp 111 y ss; Gaitán-Manfred, 2011, p 122). Es más, en puridad ya no podrá hablarse de alumnos, de individuos sometidos a unas mismas normas y a unos mismos principios, sino de sujetos considerados, tutelados y valorados en sus particulares contextos de acción e interacción, en su propia individualidad, que habrá de ser suscitada, motivada y desarrollada en todo momento (Dubet, 2006, pp 86 y ss; Le Goff, 1999, pp 46 y ss; Gauchet, 2002, p 133). Se habría así transitado de un programa que trataba de socializar al individuo por la escuela a otro cuyo objetivo será socializarlo en la escuela (Gauchet, 2002, p 120). En este contexto se inscriben algunos de los actuales discursos educativos centrados en la autonomía del niño, en el desarrollo de sus 
potencialidades y de sus capacidades. Este hecho es observable en las leyes educativas de muchos países occidentales, tales como España (véase la LOGSE especialmente, pero también la LOE), Francia (Le Goff, 1999, pp 45 y ss), Suecia o Dinamarca (Enkvist, 2000, pp 60 y ss), por citar sólo algunos ejemplos.

No obstante, este discurso que otorga un especial protagonismo al niño ha sido favorecido también, como antes se ha visto, por una nueva concepción de los saberes amparada por los poderes públicos, que se articula fundamentalmente entorno al concepto de competencias, y que focaliza extraordinariamente su atención sobre los alumnos y sus respectivos contextos de aprendizaje (RopéTanguy, 1994, p 241; Le Goff, 1999, p 54; Bolivar, 2008, p 13; Laval, 2004, p 99; Rychen y Salganik, 2004). En este contexto se ha primado la autonomía del alumno. Por autonomía se entiende desde esta perspectiva que los estudiantes pongan en práctica su capacidad de aprender en situaciones diversas, que sean ellos mismos los que indaguen e investiguen, y que incluso se atrevan a construir los contenidos de su propio aprendizaje (Pérez Gómez, 2008, p 90). En esta línea el proyecto DeSeCo, elaborado por la OCDE en el año 2000, considera precisamente que la autonomía debe ser una de las competencias clave (Rychen y Salganik, 2004), y señala el camino para su implantación en todos los sistemas educativos de su área (Gimeno Sacristán, 2008, pp 31 y ss).

En suma, las instituciones educativas han visto como se transformaba su cometido en un escenario en el que los alumnos adquirían un mayor protagonismo, y en el que los saberes tenían una orientación más utilitaria y práctica, correspondiendo al alumno un papel más activo en el aprendizaje.

En este contexto se ha modificado también el carácter y el contenido de la función docente en varios sentidos.

\section{3.- La nueva función de los enseñantes}

El tradicional rol docente, legitimado por unos saberes sobre los que existía un gran consenso, que conferían a sus portadores dignidad y respeto, y que eran transmitidos a los alumnos como parte de su proceso de desarrollo personal en el marco de una sociedad meritocrática y democrática articulada entorno a las clases, se ha transformado en los nuevos escenarios educativos. Esta transformación está relacionada tanto con la nueva concepción de los saberes como con el estatuto que han adquirido los alumnos en los actuales sistemas educativos.

En efecto, en los últimos años, tal como se ha visto, ha emergido una concepción de la educación cada vez menos academicista, orientada más al hacer que al conocer y valorada ante todo por su operatividad práctica en determinados contextos. Como dice la OCDE en un Informe ya citado, "los jóvenes que abandonan la escuela tienen necesidad de una gama de cualificaciones y de competencias que sólo en parte se adquieren en un programa tradicional de estudios" (...) "Estas cualidades son al menos tan importantes como los conocimientos técnicos o como los propios de una 
determinada disciplina". En suma, es más importante, "la capacidad de aprender a lo largo de la vida que la adquisición de los conocimientos de una determinada disciplina" (OCDE, 1998, pp 25-39).

En estas circunstancias ha cambiado la percepción de la función docente. Su finalidad principal ya no será la transmisión de conocimientos, pues éstos cambian permanentemente a medida que lo hacen también los escenarios en los que éstos han de ser aplicados, sino la de proporcionar a los estudiantes las habilidades y las actitudes que demandan los nuevos ámbitos laborales. Para ello ya no bastará con el dominio de los saberes relativos a un campo académico determinado. "No se puede contar sólo con la formación inicial para preparar completamente a los enseñantes- se dice desde la OCDE-, en particular en un contexto social, económico y educativo que se modifica rápidamente (...) Esta formación debe ser completada por una formación continua y por un perfeccionamiento profesional" (...) Perfeccionamiento que "será- indica el informe- una prioridad de los gobiernos para poner en práctica las reformas educativas" (OCDE, 1998, pp 17-31).

El estatuto docente cambia también en un contexto educativo como el actual en el que los alumnos gozan de mayor participación y autonomía, y en el que son invitados a ser los protagonistas de su aprendizaje. En un escenario como este, la función de los profesores no ha de ser tanto la de transmitir conocimientos como la de actuar como una especie de tutores o de guías que acompañen al alumno en su propio proceso de aprendizaje (véase Memorandum de la Comisión Europea, 2000; citado en Laval, 2004, p 92; Pérez Gómez, 2008, p 95)

Se instaura así un nuevo profesionalismo docente más técnico- importan más los métodos y los procedimientos que los contenidos-; más individualista- cada uno debe probar su competencia-, y continuamente revisable- la formación no es gradual ni acumulativa, sino un proceso en continua mutación, en el que se valoran más las destrezas, el cómo aprender y transmitir el nuevo conocimiento, que el contenido de lo que se aprende y de lo que posteriormente ha de ser enseñado. Este profesionalismo ha sido, como se ha visto, especialmente potenciado por las principales organizaciones políticas internacionales, y como tal es parte esencial de los discursos legítimamente proclamados por estas instituciones, que se proponen como auténticos programas de acción para los distintos Estados. "El perfeccionamiento profesional de los enseñantes- se dice desde la OCDE- será una prioridad de los gobiernos para poner en práctica las reformas educativas" (OCDE, 1998, p 31).

Muchos Estados, de hecho, han puesto en marcha programas de formación del profesorado que obedecen a estos mismos principios. Es el caso de Alemania, Reino Unido, Irlanda, Suecia, Suiza, EE.UU, Japón (OCDE, 1998: 67 y ss), Francia (Le Goff, 1999, pp 37 y ss) o España (Gimeno Sacristán, 2008), por citar sólo algunos ejemplos.

Esta nueva concepción de la profesión docente tiene además otras implicaciones. En efecto, en la medida en que despoja al enseñante de muchos de los elementos que cimentaban su legitimidad y su prestigio, esto es, la 
posesión de un saber reconocido institucionalmente del que sus discípulos carecen, ha contribuido también a erosionar su autoridad. Dicho de otro modo, si las instituciones políticas ya no amparan un proyecto común universal, como el que rigió a lo largo de la primera modernidad y en nombre del cual hablaba el maestro, los fundamentos de su autoridad se pierden irremediablemente. En este contexto encuentra hoy explicación los déficit de autoridad que padecen muchas instituciones educativas, y que no son sino otra manifestación más de su debilidad institucional.

Este declive institucional (Dubet, 2006) no ha sido causado únicamente por la acción de los poderes públicos. En este hecho han intervenido muchos otros factores. Pero estos poderes con sus discursos, sus programas y sus líneas de acción han contribuido sustancialmente a tal causa.

En efecto, tal como hemos expuesto en este artículo, ellos han favorecido una concepción del saber puramente operativa e instrumental, que cobraba sentido únicamente por su funcionalidad en los distintos contextos productivos, y no en relación con una determinada tradición académica o escolar.

También ellos han promovido especialmente la aplicación al ámbito educativo de muchas de las normas y de los principios que rigen en el espacio público democrático, transformando al alumno en un verdadero ciudadano. Un alumno que se ha convertido asimismo en el verdadero protagonista de los procesos de enseñanza aprendizaje.

Finalmente, al impulsar procesos de formación continúa en distintas habilidades y técnicas, las instituciones políticas han cuestionado a los docentes en lo que más los definía, a saber, la posesión de un determinado saber adquirido a lo largo de los años que los capacitaba para ser los dignos portavoces de la institución en nombre de la que hablaban. Este saber se ha vuelto cambiante y los profesores ya no son sus principales y legítimos transmisores.

Todos estos fenómenos están directamente relacionados con la desinstitucionalización del ámbito escolar, y todo ellos han sido favorecidos, cuando no directamente promovidos, por la acción de los poderes públicos.

Esta desinstitucionalización ha desembocado así en un escenario completamente distinto al que habían creado y conformado las ideologías políticas modernas, para las que la escuela era una especie de santuario laico destinado a formar a todos los individuos en unos mismos principios universales, convirtiendo a cada uno de ellos en personas autónomas y libres.

Este proyecto así formulado era el resultado del programa civilizador de la modernidad (Elías, 1993), que en esencia consistía en formar individuos racionales y libres, conscientes de sus deberes para con la sociedad; individuos, en una palabra, civilizados. Este programa, que incluía la socialización y la individualización en un mismo acto (Dubet, 2006, pp 48 y ss), y que había sido impulsado por los Estados Modernos, es el que ahora se viene abajo por la misma acción de los poderes que siglos atrás lo habían 
conformado. En efecto, cuando desaparece una cultura común en la que los Estados y los individuos se reconocen; cuando en lugar de esa cultura emergen saberes instrumentales; cuando los sujetos ya no se definen por una pertenencia común, sino por sus distintas particularidades; cuando los portavoces de estas instituciones tienen cada vez más dificultades para dirigirse a sus miembros en nombre de criterios universales. Entonces es cuando ese proyecto civilizatorio más se resiente y resulta cada vez más difícil invocarlo para continuar educando.

\section{CONCLUSIÓN}

A lo largo del presente artículo hemos querido mostrar una de las vertientes de la crisis educativa actual, la que se vincula más directamente con los discursos y las acciones de las instituciones políticas nacionales e internacionales. Desde el punto de vista que aquí se sostiene esta crisis no es meramente coyuntural, por cuanto se relaciona con el agotamiento del proyecto civilizatorio moderno, tal como fuera concebido y formulado desde los inicios de la Modernidad. Proyecto para el que la educación era uno de sus pilares fundamentales.

De acuerdo con dicho programa, la educación era uno de los principales medios para socializar e integrar a los individuos para constituirlos así en sujetos orgullosos de su pertenencia, pero también de su autonomía y de su independencia. Esta doble condición, de individuos integrados y a la vez autónomos, se lograba por medio de su socialización en un mismo universo de normas y de principios, que constituían la esencia del programa civilizatorio moderno, amparado e impulsado por el Estado.

Este proyecto civilizatorio se anclaba en una serie de principios. En un primer momento- desde finales del siglo XIV hasta la quiebra del Antiguo Régimenrespondió a la voluntad del Estado Moderno de civilizar, esto es, de domesticar a las élites poniéndolas bajo su control o directamente a su servicio. Todo lo cual se tradujo en un programa educativo que contenía los principios esenciales de este proyecto civilizatorio, que debía ser inculcado mediante formas disciplinarias basadas en la persuasión y el autocontrol, con el fin de producir conciencias civilizadas.

La llustración y el Liberalismo continuaron este proyecto, aunque con un acento más productivista y utilitario, y también con un mayor afán por extenderlo a todas las capas sociales. No obstante, siguió vivo el deseo de civilizar, de introducir a todos los individuos en un mismo universo de valores y de normas, aunque fuese para crear trabajadores y ciudadanos, personas conscientes de sus deberes productivos y patrióticos, pero también imbuidas de los principios constitutivos de una sociedad culta, libre y abierta. Los distintos saberes escolares tenían por finalidad, en un contexto como este, integrar a los individuos en los lugares que el nuevo orden social exigía, pero también liberarlos de sus condicionamientos sociales, haciéndolos de este modo más autónomos y más libres. 
Los contenidos esenciales de este programa fueron llevados a la práctica por los distintos Estados a lo largo del siglo XX, hasta completar la universalización de la enseñanza, al menos hasta las primeras etapas de la educación secundaria. Es el momento en el que el proyecto moderno alcanza su plenitud, el momento de su mayor encanto, cuando la educación se convierte en un instrumento esencial para lograr el progreso económico y social de todos los individuos en una sociedad libre, abierta y democrática. Y como tal contribuyó sustancialmente a la legitimación de los Estados que lo impulsaron.

Pero esta plenitud poco duró. Pronto aparecieron las primeras críticas que tornaron el encanto en desencanto. Primero, se dijo que la educación mantenía, y que incluso favorecía las desigualdades sociales, y que además tenía un carácter represivo y autoritario. Más tarde, que era incapaz de impulsar el progreso social y el económico. Todas estas críticas fueron debilitando el programa educativo moderno.

La respuesta a esta situación fue una reformulación del programa republicano en unos términos que contribuyeron todavía más a erosionarlo, por cuanto favorecieron un proceso en el que los principios en los que se basaba este programa civilizatorio se pusieron en cuestión desde varios frentes.

En primer lugar, en todo lo relacionado con el contenido de los saberes. Si en un principio se trataba de educar a todos los individuos en ellos con el fin de promoverlos individual y socialmente. Más tarde, en el contexto socioeconómico surgido a mediados de los años setenta, se pondrá en cuestión la capacidad de estos saberes para realizar este cometido, por lo que las instancias políticas nacionales e internacionales se propondrán amoldarlos a las exigencias de la economía. Por esta vía los saberes adquirirán un carácter más fragmentario, utilitario y práctico, perdiendo su conexión con una determinada tradición enraizada en el proyecto educativo moderno. En segundo lugar, los alumnos adquirirán un mayor protagonismo, tanto en relación con una concepción de la educación más enfocada al aprendizaje que a la enseñanza, como con el papel que se les atribuirá en unos escenarios educativos configurados a semejanza del espacio público democrático, y en el que cada sujeto será tratado conforme a sus circunstancias particulares.

Como consecuencia de todo ello, los profesores ya no aparecerán en los discursos y en los programas educativos de los Estados como los portavoces de un conocimiento que ya no monopolizan ni encarnan. Serán los poderes políticos y económicos, por un lado, y los discursos psicológicos y pedagógicos, por el otro, los que legitimarán y determinarán cual ha de ser su contenido. Como dichos saberes tendrán que adaptarse continuamente a las circunstancias cambiantes del mercado y a las de los individuos que aprenden, la cualificación que se demanda a los docentes, su competencia, será mucho más metodológica y práctica que de carácter académico. Pero como estas competencias varían en función del contexto al que se apliquen, su actualización tendrá que ser permanente, lo que incide todavía más en la descualificación docente. 
Se perturba así lo que era la esencia del proyecto educativo moderno. Este proyecto ya no se incardina en una tradición civilizadora en la que se reconocían todos sus miembros. Por el contrario, esta tradición se fragmenta, se hace añicos, cuando se transforma para remitirse a los contextos cambiantes de la economía, o a las particularidades de cada individuo. Es entonces cuando las máscaras de la civilidad caen (Sennett, 2011, p 325), y en su lugar emergen unas relaciones muchos más individualizadas y desinstitucionalizadas, en las que unos actores, los alumnos, actúan exhibiendo su personalidad en cada momento, reclamando un mayor protagonismo en cuanto sujetos; otros, los educadores, tratan de justificar sus acciones permanentemente, poniendo en juego sus capacidades personales, sus competencias, incapaces de recurrir ya a principios aceptados comúnmente; y los Estados, con su intervención, acentúan todavía más esta tendencia cuando asignan a las instituciones fines instrumentales, disuelven la profesión en un conjunto de técnicas y de métodos al servicio de aquellos fines, y convierten a los alumnos en los protagonistas de su propio aprendizaje, y en sujetos de una ciudadanía sin contenido.

\section{BIBLIOGRAFÍA}

(1994) ALALUF, Marcelle-STROOBANTS, Mateo “¿Moviliza la competencia al obrero?”. Revista Europea de Formación Profesional, 1, 46-55, Madrid, pp 46-55

(1987) ALIGHIERO MANACORDA, Mario, Historia de la educación (Vol II) Ed.SXXI, México

(2005) ALONSO, Luís Enrique, La era del consumo. Ed. SXXI. Madrid.

(1986) AMELANG, James S, La formación de una clase dirigente: Barcelona 1490-1714. Ed. Ariel, Barcelona

(2003) ARENDT, Hannah, “¿La crisis en la educación?”. En $\mathrm{H}$ Arendt, Entre el pasado y el futuro, Península, Barcelona, pp 269-301

(1989) ARIÉS, Philippe, El niño y la vida familiar en el Antiguo. Ed Taurus, Madrid

(1974) ALTHUSSER, Louis, La filosofía como arma de la revolución. Ed. SXXI. México

(1975) BAUDELOT, CH-ESTABLET, R, La escuela capitalista en Francia Ed. SXXI, Madrid

(2007) BAUMAN, Z, Los retos de la educación en la modernidad líquida, Ed. Gedisa, Barcelona.

(2006) BECK, Ulrich, La sociedad del riesgo, Ed. Paidós, Barcelona.

(1989) BERNSTEIN, Basil Clases, códigos y control. Ed. Akal, Madrid

(2008) BOLIVAR, Antonio, El discurso de las competencias en España: educación básica y educación superior, en Revista de Docencia Universitaria, n²2, pp 123

(2002) BOLTANSKI, Luc y CHIAPELLO, Ève, El nuevo espíritu del capitalismo, Ed.Akal, Madrid

(2001) BOURDIEU, Pierre-PASSERON, Jean Claude, La reproducción. Elementos para una teoría del sistema de enseñanza. Ed. Popular, Madrid

(1985) BOWEN, James Historia de la educación Occidental (Vol. III) Ed. Herder, Barcelona 
(1994) CASTIGLIONI, Baldassare El cortesano. Madrid: Ed. Cátedra

(2003) COMISIÓN DE LAS COMUNIDADES EUROPEAS. Libro Blanco. Crecimiento competitividad y empleo. Retos y pistas para entrar en el siglo XXI, Ed. Oficina de publicaciones Oficiales de las Comunidades Europeas, Bruselas.

(1985) COOMBS, Philip Hall, La crisis mundial en la educación. Perspectivas actuales. Madrid: Ed. Santillana

(2006) DUBET, François, El declive de la institución. Profesiones, sujetos e individuos ante la reforma del Estado, Ed. Gedisa, Barcelona

(2011) DURÁN VÁZQUEZ, José Francisco, La metamorfosis de la ética del trabajo. Constitución, crisis y reconfiguración de la ética del trabajo en la modernidad tardía, Ed. Andavira, Santiago de Compostela.

(1992) DURKHEIM, Émile Historia de la educación y de las doctrinas pedagógicas, Ed. La Piqueta, Madrid.

(1993a) ELIAS, Norbert, El proceso de civilización, Ed. FCE, Madrid

(1993b) ELIAS, N La sociedad cortesana. Madrid: Ed. FCE, Madrid.

(2000) ENKVIST, Inger, La educación en peligro. La pedagogía progresista y el declive de nuestro sistema educativo. Ed. Unisón Producciones, Madrid

(1985) ERASMO De la urbanidad en las maneras de los niños. Ed. Artegraf, Madrid

(2001) FERNÁNDEZ ENGUITA Mariano, Educar en tiempos inciertos. Ed. Morata, Madrid

(1989) FERNÁNDEZ ENGUITA, LEVIN, Henry M "Las reformas comprensivas en Europa y las nuevas formas de desigualdad educativa", en Revista de Educación, 289, Madrid, pp 49-64, Madrid

(1999) FOUCAULT, Michel Vigilar y castigar. Ed. Círculo de lectores, Barcelona:

(2011) GAITÁN, Lourdes-LIBEL, Manfred, Ciudadanía y derechos de participación de los niños, Ed. Síntesis, Madrid

(1999) GAITÁN, Lourdes, El espacio social de la infancia. Los niños en el Estado de Bienestar, Ed. Consejería de Sanidad y Servicios Sociales de la Comunidad de Madrid, Madrid

(2006) GAITÁN MUÑOZ, Lourdes "El bienestar social de la infancia y los derechos de los niños", en Política y Sociedad, Vol 43, oㅜ 1, Madrid, pp 63-80

(1967) GARIN, Eugene, La educación en Europa. ed. Crítica. Barcelona

(2002) GAUCHET, Marcel: “L'école à l'école d'ellemême. Contraintes et contradictions de l'individualisme démocratique", en GAUCHET, M, La démocratie contre elle-même, Ed. Gallimard, París, pp 109-169.

(1785) GENOVESI, Antonio Lecciones de comercio, o bien de economía civil (3 Vols), Madrid, Editado por la viuda de Ibarra, hijos y compañía

(2008) GIMENO SACRISTÁN, José, "Diez tesis sobre la aprente utilidad de las competencias en educación", en 
GIMENO SACRISTÁN, J, Educar por competencias ¿qué hay de nuevo?, Ed. Morata, pp 15-58, Madrid (1988) GOBERNADO ARRIBAS, Rafael "La construcción del sistema educativo meritocrático", en Revista española de pedagogía, 180, pp 306-317, Madrid

(1972) GUTIERREZ ZULUAGA, Isabel Historia de la Educación. Ed. Narcea, Madrid

(1999) HABERMAS, Jürgen, Problemas de legitimación en el capitalismo tardío, Ed. Cátedra, Madrid.

(2008) JONES, Lyn-MOORE, Rob, "La apropiación del significado de competencia: el movimiento de la competencia, la Nueva Derecha y el proyecto del "cambio cultural", en Revista de curriculum y formación del profesorado, 12, 3, pp 1-20

(1981) JULIA, Dominique, Les trois coleurs du tableau noir. La Révolution. Ed. Belin, París

(1999) LASH, Christopher La cultura del narcisismo. Ed. Andrés Bello, Barcelona

(2004) LAVAL, Christian, La escuela no es una empresa. El ataque neoliberal a la enseñanza pública, Ed. La Découverte, París

(2002) LE GOFF, Jean-Pierre, La démocratie posttotalitaire, Ed. La Découverte, París

(1999) LE GOFF, Jean-Pierre, La barabarie douce. La modernisation aveugle des entreprises et de l'école, Ed. La Découverte, París

(1996) LE GOFF, J.P, «Formation, école, entreprise: confusion et enjeux», en ANDRÉANI, Tony-GAUDEAUX, Jean-François-NAUD, Didier (dirs.) L'entreprise: Lieu de nouveaux contrats?, Ed. L'Harmattan, pp 285-295, París (1994) LUZURIAGA, Lorenzo Historia de la educación y de la pedagogía. Ed. Losada, Buenos Aires

(1987) MANACORDA, Mario Alighiero Historia de la educación, Ed. SXXI, México.

(1991) MANN, Michael Las fuentes del poder social (Vol I) Alianza editorial, Madrid

(1998) MARSHALL, T.H-BOTTOMORE, Tom Ciudadanía y clase socia, IAlianza Editorial. Madrid (2005) MARTINEZ BONAFÉ, Jaume, La formación del profesorado y el discurso de las competencias, en Revista Interuniversitaria de Formación del Profesorado, 18 (3), 127-144

(1981) MEYER, Philippe, El niño y la razón de Estado, Ed. Zero, Madrid

(1999) MIGUELEZ, Faustino-PRIETO, Carlos (dir y coord.), Las relaciones de empleo en España, Ed. SXXI, Madrid

(1970) NEIL S, Alexander, Libres enfants de Summerhill, Ed. François Maspero, París.

(2000) OCDE, Societé du savoir et gestions des connaissances, enseignement et compétences, OCDE, París

(1998) OCDE, L'école à la page. Formation continue et perfectionnement professionnel des enseignantes, OCDE, París

(1992) OCDE, Écoles et entreprises: Un nouveau partenariat, OCDE, París 
(1991) OCDE, Escuelas y calidad de la enseñanza, MEC, Madrid

(1993) ORTEGA, Félix "La crisis de los sistemas escolares", en GARCÍA DE LEÓN, María A-DE LA FUENTE, Gloria-ORETGA, Félix (eds.) Sociología de la educación, Ed. Barcanova, Barcelona

(1992) OZOUF, Jacques-OZOUF, Mona, La republique des instituteurs. Ed. Gallimard, París

(1984) OZOUF, Mona, L'école de la France. Éssais sur la Révolution, l'utopie et l'enseignement. Ed. Gallimard, París

(2008) PÉREZ GÓMEZ, Ángel, “¿Competencias o pensamiento práctico? La construcción de los significados de representación y de acción", en GIMENO SACRISTÁN, José, Educar por competencias ¿qué hay de nuevo?, Ed. Morata, pp 59-102, Madrid

(1999) PICÓ, Josep, Cultura y modernidad. Seducciones y desengaños de la cultura moderna, Ed. Alianza Editorial, Madrid

(1990) PIORE, Michael J y SABEL, Charles F, La segunda ruptura industrial. Alianza Editorial, Madrid

(2005) PRATS, Joaquim-REVENTÓS, Francesc (dir.) Los sistemas educativos europeos ¿Crisis 0 transformación? Ed. Fundación La Caixa, Barcelona:

(1967) PROST, Antoine Histoire de l'ensegnement en France, 1800-1967, Ed. Armand Colin, París

(1980) PUELLES BENITEZ, Manuel, Educación e ideología en la España contemporánea. Ed. Labor, Barcelona

(1993) PUELLES BENITEZ, M, «Estado y educación en las sociedades europeas", en Revista Iberoamericana de Educación, №1, Enero-Abril 1993

(1983) RAYNAUD, Philippe "L'idéologie réformatrice ou le consensus éducatif", en Pouvoirs, 30, pp 113-123, París

(2002) RENAUT, Alain, La libération des enfants. Contribution philosophique à une histoire de l'enfance, Ed. Calmann-Lévy Bayard, París

(2004) RYCHEN, D.S. Y SALGANIK, L.H. (eds.) Definir y seleccionar las competencias fundamentales para la vida. Ed. FCE, México

(1988) RODRIGUEZ, Jorge, "Crisis " del Estado del Bienestar y política educativa. Arbor, 514, pp 99-120, Barcelona

(2007) RODRIGUEZ PASCUAL, Iván, Para una sociología de la infancia: aspectos teóricos y metodológicos, Ed. CIS, Madrid

(1994) ROPÉ, François-TANGUY, Lucie, Savoirs et compétences. De l'usage de ces notions dans l'école et l'entreprise, Ed. L'Harmattan, París

(1970) RUIZ BERRIO, Julio, Política escolar de España en el siglo XIX, Ed. CIS, Madrid

(2011) SENNETT, Richard, El declive del hombre público, Ed. Anagrama, Barcelona

(2009) SERRANO PASCUAL, Amparo, "Regulación supranacional y despolitización del trabajo: el caso del paradigma de la activación", en CRESPO, EduardoPRIETO, Carlos-SERRANO, A, Trabajo, subjetividad y ciudadanía. Paradojas del empleo en una sociedad en transformación, Ed. CIS, pp 259-289, Madrid. 
(2002) SERRANO PASCUAL, A-CRESPO, E, El discurso de la Unión Europea sobre la sociedad del conocimiento, en Revista española de Investigaciones Sociológicas, Madrid, no 97, pp 189-207, ISSN 0210-5233, Madrid

(2000) SERRANO PASCUAL, A "EI concepto de empleabilidad en la estrategia europea de lucha contra el desempleo: una perspectiva crítica", en Revista del Ministerio de Trabajo y Asuntos Sociales, 21, pp137-149 Madrid

(2006) TAYLOR, Charles, Imaginarios sociales modernos, Ed. Paidós, Barcelona

(1999) TERREN, Eduardo, Educación y modernidad. Entre la utopía y la burocracia. Ed. Anthropos, Barcelona (1993) THERBORN, Göran, "Los derechos de los niños desde la constitución del concepto moderno del menor: un estudio comparado de los países occidentales", en MORENO, Luís (comp.) Intercambio social $y$ desarrollo del bienestar, Ed. CIS, Madrid TORRES SANTOMÉ, Jurjo (2008) "Obviando el debate sobre la cultura en el sistema educativo: cómo ser competentes sin conocimientos", en GIMENO SACRISTÁN, José, Educar por competencias ¿qué hay de nuevo?, pp 143-175 Madrid: Morata

(1989) VARELA, Julia-ALVAREZ URÍA, Fernando Arqueología de la escuela. Ed. La Piqueta, Madrid (1991) VARELA, Julia "El triunfo de las pedagogías psicológicas", en Cuadernos de Pedagogía, 198, Madrid, pp 56-58

(1988) WILLIS, Paul, Aprendiendo a trabajar. Cómo los chicos de clase obrera consiguen trabajos de clase obrera. Ed. Akal, Madrid:

\section{NOTAS FINALES}

\footnotetext{
${ }^{1}$ Prusia fue el primer Estado que estableció un sistema educativo nacional en 1810, sistema en el que se basó el Estado alemán para crear a finales de siglo un sistema de educación estatal y universal. En Francia, la centralización educativa comenzó en época napoleónica, pero serán las reformas de Jules Ferry (1880-1883) las que establecerán la escuela laica, gratuita y obligatoria. En Inglaterra, en 1870, el gobierno liberal de Gladstone, a través del ministro Foster, inicia el proceso de universalización de la enseñanza, que fue completado más tarde, en 1876, por Disraeli, que estableció la obligatoriedad escolar. En España la ley Moyano establece en 1857 la universalización y la obligatoriedad de la enseñanza. Pero es en la II República (1933) cuando se crea un sistema de educación universal, estatal y obligatorio. En EE.UU la escuela pública quedó instaurada entorno a 1850. En América del Sur, las recién creadas repúblicas van instaurando los distintos sistemas de educación nacional a lo largo de todo el siglo XIX (Bowen, 1985 III: 335 y ss; Luzuriaga, 1994: 181 y ss; Prost, 1967).

2 EE.UU fue el país más precoz en la adopción de medidas de este tipo, logrando una escolarización del $42 \%$ en educación secundaria en 1940. En Inglaterra fue el Acta de educación de 1944 la que estableció un sistema nacional de educación secundaria. En Francia, el Plan Longevin-Wallon de 1947 establecerá un sistema de educación secundaria nacional para todo el país (Bowen, 1985: 554 y ss)

${ }^{3}$ El crecimiento del gasto educativo en la década de los años 50 y 60 fue más que notable. En el conjunto de los países de la OCDE dicho gasto fue del 10\% anual, equivalente al doble del crecimiento del PNB (Prost, 1967: 487; Gobernado, 1988)
} 


\footnotetext{
${ }^{4}$ No obstante, la extensión de la obligatoriedad escolar no tuvo la misma intensidad en todos los lugares. Los países Escandinavos fueron los que más avanzaron en este sentido con las denominadas reformas comprensivas emprendidas en los años 50, que en la práctica significaban la ampliación de la obligatoriedad escolar y la integración de todos los alumnos en la escuela única. El Reino Unido siguió este camino en los años 60 , aunque de una forma más tímida. Otros países consumaron estas reformas más tarde. Italia lo hizo en 1972; Francia amplió la escolarización hasta los 16 años en 1959, durante la V República. Los países del sur de Europa establecieron una escolarización obligatoria más modesta. En el caso de España, la Ley Villar Palasí de 1970 creó la Educación General Básica como un sistema de enseñanza universal y obligatoria hasta los 13 años.

${ }^{5} \mathrm{El}$ agotamiento del sistema económico fordista era un hecho evidente en la mayoría de las economías desarrolladas desde mediados de los años 70 del pasado siglo (Piore y Sabel, 1990), cuando la producción en masa ya no pudo ser absorbida por una población saturada de bienes de consumo duraderos. Todo lo cual dio lugar a la emergencia de un nuevo modelo productivo, conocido como postfordista, más intensivo en tecnología y menos en mano de obra, que produjo un incremento notable de las tasas de desempleo y de precariedad laboral en la mayoría de los países de la OCDE en la década de los 80 y los 90 (OCDE, I 2000: 27 y 41).

${ }^{6} \mathrm{~A}$ este respecto merece se destacado el Informe Reagan, "A nation at risk". En él se decía que los problemas económicos de los EE.UU se derivaban en buena medida de las deficiencias de su sistema educativo (Morgenstern, 1987). En Inglaterra las reformas emprendidas por el gobierno Thatcher a comienzos de la década de los 80 iban encaminadas a conseguir una mayor efectividad y eficiencia, para lograr así una mejor adaptación de la educación a los requerimientos del sistema económico y del mercado laboral (Prats-Reventós, 2005: 69)

${ }^{7}$ Las reformas llevadas a cabo en Francia y en España entre finales de los años 80 y comienzos de los 90 se inscriben en esta perspectiva. En realidad, Francia ya había llevado a cabo una reforma de este tipo con la ley Haby de 1975 que instituyó el "Collège Unique" (Raynaud, 1983: 115, Prats-Reventós, 2005: 32). Las reformas de los años 80 avanzaron en este mismo sentido (Le Goff, 1999: 37 y ss). Los países del sur de Europa, como España promulgaron estas leyes entre finales de los años 80 y comienzos de los 90 (LOGSE, 1990)
} 